\title{
Fragmentation and shear band formation by slow compression of brittle porous media
}

\author{
Gergő Pál, ${ }^{1}$ Zoltán Jánosi, ${ }^{2}$ Ferenc Kun, ${ }^{1,2, *}$ and Ian G. Main ${ }^{3}$ \\ ${ }^{1}$ Institute for Nuclear Research, Hungarian Academy of Sciences (Atomki), P.O. Box 51, H-4001 Debrecen, Hungary \\ ${ }^{2}$ Department of Theoretical Physics, University of Debrecen, P.O. Box 5, H-4010 Debrecen, Hungary \\ ${ }^{3}$ School of Geosciences, University of Edinburgh, EH9 3 FE Edinburgh, United Kingdom
}

(Received 29 June 2016; published 16 November 2016)

\begin{abstract}
Localized fragmentation is an important phenomenon associated with the formation of shear bands and faults in granular media. It can be studied by empirical observation, by laboratory experiment, or by numerical simulation. Here we investigate the spatial structure and statistics of fragmentation using discrete element simulations of the strain-controlled uniaxial compression of cylindrical samples of different finite size. As the system approaches failure, damage localizes in a narrow shear band or synthetic fault "gouge" containing a large number of poorly sorted noncohesive fragments on a broad bandwidth of scales, with properties similar to those of natural and experimental faults. We determine the position and orientation of the central fault plane, the width of the shear band, and the spatial and mass distribution of fragments. The relative width of the shear band decreases as a power law of the system size, and the probability distribution of the angle of the central fault plane converges to around 30 degrees, representing an internal coefficient of friction of 0.7 or so. The mass of fragments is power law distributed, with an exponent that does not depend on scale, and is near that inferred for experimental and natural fault gouges. The fragments are in general angular, with a clear self-affine geometry. The consistency of this model with experimental and field results confirms the critical roles of preexisting heterogeneity, elastic interactions, and finite system size to grain size ratio on the development of shear bands and faults in porous media.
\end{abstract}

DOI: 10.1103/PhysRevE.94.053003

\section{INTRODUCTION}

Shear bands are zones of highly localized strain that develop spontaneously during the deformation of a wide range of materials, including alloys, metals, plastics, and polymers which deform by thermally activated plastic mechanisms [1-3]. They can also occur by locally brittle failure in disordered granular media such as concrete, ice, rock, and some ceramics, where the microscopic failure mechanism involves tensile or shear fracture [4,5]. In particular, natural faults and localized shear bands in brittle porous media often contain a loose aggregate of fragments known as a "fault gouge." These fragments are created by subsequent fracturing, crushing, and milling during the relative motion of the two intact bodies on either side of the shear band or fault [6-9]. The frictional properties and stability of faults strongly depend on the shape and size of the fragments, so understanding the process of quasistatic fragmentation is extremely important in developing models for these emergent properties $[6,10]$. The evolution of the gouge with the accumulated shear can be studied on laboratory scales by shearing granular assemblies under compression. Both field measurements and laboratory experiments have confirmed that gouge particles undergo subsequent breakups and the resulting size distribution of fragments evolves to a power law [11]. Fragments of mature shear bands are typically characterized by a power law exponent of around $2.6[6,10]$.

In order to explain the power law distribution of fragments, a stochastic fragmentation model was introduced where scale free behavior was ensured by the size independent breakup probability of pieces $[6,12]$. The model was able to reproduce the size distribution of fragments under quite generic condi-

\footnotetext{
*ferenc.kun@science.unideb.hu
}

tions. More advanced approaches were worked out by simulating the breakup of grains in sheared granular assemblies, where stochastic breaking rules were set for structureless particles [13]. The drawback of the approach is that mass conservation is difficult to ensure and the results depend on the imposed breaking rules. The most realistic numerical studies of fault gouge formation were performed in the framework of discrete element models where macroscopic particles were assembled of spheres or polyhedra glued together with breakable cohesive contacts [14-16]. Computer simulation of shear cells resulted in power law size distributions of fragments with exponents close to the experimental values.

The fault gouge is not the only signature of local brittle failure associated with the localization of deformation during fault formation and slip. Most faults in nature are surrounded by a damage zone consisting of more distributed brittle structures such as smaller splays, branches, subfaults, and microcracks [17]. The fault itself develops as a localized shear band formed by multiple fracturing and refracturing both prior to and during slip. To study this problem, recently we have introduced a discrete element model of porous sedimentary rocks which captures the essential ingredients of the materials' microstructure and of the dynamics of breaking [18-20].

In this paper we use our discrete element model to study the fragmentation process of slowly-compressed cylindrical samples. While damage can occur anywhere in the sample, we concentrate on the properties of the emergent shear band itself, taken to be a zone of contiguous fragmentation caused by initial fragmentation during the initial localization of deformation, and subsequent comminution and wear during shear itself. First, based on the spatial distribution of fragments we determine the orientation and width of the shear band. Using finite size scaling we demonstrate that the shear band sharpens with increasing sample size to grain size ratio, and 
its orientation angle with respect to the load direction tends to the value measured experimentally for brittle rocks, implying a coefficient of internal friction of around 0.7 or so. The size distribution of fragments has a power law behavior with an exponent close to the measured values. Simulations revealed that the compressive loading leads to a self-affine character of fragment shapes and it also gives rise to a higher value of the mass distribution exponent compared to shock fragmentation.

\section{SIMULATION OF UNIAXIAL COMPRESSION}

Our discrete element model (DEM) has recently been introduced to investigate the compressive failure of heterogeneous materials $[18,19]$. In the model porous rock samples are reconstructed by simulating the sedimentation process of grains represented by spherical particles. Discrete element simulations were performed by sedimenting particles one-byone inside a cylindrical container under the action of gravity [18]. Particles suffered several collisions with the particles of the growing sediment layer and with the container wall until their final position was found. The particle radius $R$ was sampled from a log-normal distribution which describes reasonably well the statistics of grain sizes in earth materials such as sandstone in the regime of large particles [17]. The range of particle size $R_{\min } \leqslant R \leqslant R_{\max }$ is limited in the algorithm since very small particles may settle to the bottom of the container bouncing through the void space between the large ones. To avoid this size segregation, the ratio of the largest $R_{\max }$ and smallest $R_{\min }$ radii was set to $R_{\max } / R_{\min }=20$, while the average particle radius $\bar{R}$ was fixed to the value $\bar{R}=5 R_{\min }$ for all the samples considered [18].

Cohesion is introduced in such a way that the particles are coupled by beam elements along the edges of Delaunay triangles constructed with the particle centers. The length and cross section of beams are determined by the distance of the coupled particles and by their radii, respectively, which implies disorder in the physical parameters of beams. Under mechanical load beams gradually deform and break according to a physical breaking rule when overstressed:

$$
\left(\frac{\varepsilon_{i j}}{\varepsilon_{\mathrm{th}}}\right)^{2}+\frac{\max \left(\left|\Theta_{i}\right|,\left|\Theta_{j}\right|\right)}{\Theta_{\mathrm{th}}} \geqslant 1 .
$$

Here $\varepsilon_{i j}$ denotes the axial strain of the beam between particles $i$ and $j$, while $\Theta_{i}$ and $\Theta_{j}$ are the rotation angles of the two beam ends [18,21]. The first and second terms of the breaking rule Eq. (1) represent the contribution of stretching and bending (shear of the particle contacts) deformations to breaking. The breaking parameters $\varepsilon_{\text {th }}$ and $\Theta_{\text {th }}$ have constant values for all the beams $\varepsilon_{\text {th }}=0.003$ and $\Theta_{\text {th }}=2^{\circ}$, so that the random packing of grains is the only source of disorder in the system. Those particles which are not coupled by beams interact through contact forces where the normal force is modeled by a Hertz contact with viscoelastic damping [22], while for the tangential force the Coulomb friction law is implemented with the friction coefficient $0.5[22,23]$. The equation of motion of the particles is solved numerically to generate the time evolution of the system induced by external mechanical loading. Further details of the model construction and the parameter settings can be found in Refs. [18,19].
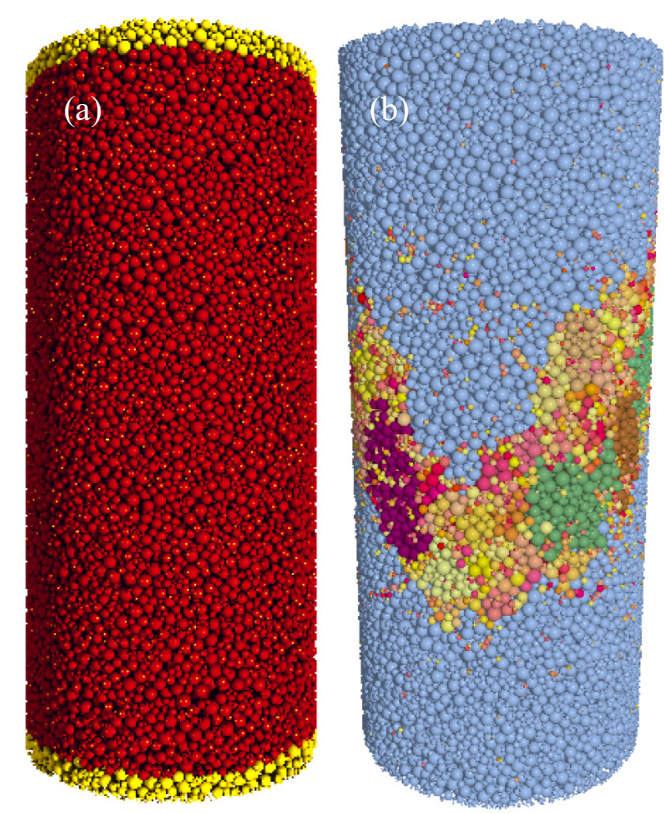

FIG. 1. (a) A cylindrical sample of $10^{5}$ particles. The clamped particle layers at the bottom and top of the sample are highlighted by yellow color. (b) Final reassembled configuration where particles are colored according to the size of fragments they belong to. The stripe of contiguous small sized fragments with a large number of different colors indicates the position of the shear band.

In the present study strain controlled uniaxial compression of cylindrical samples was simulated using the same boundary and loading conditions as in Refs. [18,19]: a few particle layers on the top and bottom of the cylinder are clamped and the top layers are moved at a constant speed while the bottom ones are kept fixed [for illustration see Fig. 1(a)]. No lateral confinement is imposed so that the side walls of the cylinder are free to move. The simulation stops when the force acting on the top layer drops down to zero. In our previous studies the model was used to investigate the time evolution of the process of compressive failure. We have demonstrated that the gradual damaging proceeds in bursts of beam breakings which are analogous to sources of acoustic emissions or crackling noise of real experiments $[18,19,24]$. The model proved to be very successful in reproducing the observed power law statistics of burst sizes, energies, durations, and of the waiting times between consecutive events [18,19]. Most recently we have also analyzed the details of the time series of bursts of the model searching for the record breaking events [20].

Here we focus on the spatial structure of damage and fault gouge formation obtained in the final state of the fracture process. Simulations were carried out with five different system sizes; i.e., samples composed of $10000,20000,50000$, 100000 , and 250000 particles were generated with a total number of repeated random samples of $642,842,360,560$, and 278, respectively. The aspect ratio defined by the diameter $D$ to height $H$ ratio of the cylinder was fixed to $H / D=2.3$ so that the factor of 25 difference in the volume implies that the linear extensions of the largest sample are $25^{1 / 3} \approx 2.92$ times greater than the ones of the smallest sample. The cylinder diameter to modal grain size ratio is $20.7,25.9,35.7,45.1$, and 
60.5 for the sample sizes considered. Since in laboratory tests the typical grain size of reservoir rocks is about $200 \mu \mathrm{m}$, the simulated samples of 250000 particles correspond to $12 \mathrm{~mm}$ sample size of real experiments; cf. the most commonly used laboratory sample size of $35 \mathrm{~cm}$. Considering different system sizes enabled us to perform finite size scaling analysis which proved to be essential for the present study and may motivate a similar analysis in experiment.

\section{SHEAR BAND}

We have shown in Refs. [18,19] that under slow compression the beginning of the failure process is dominated by the quenched structural disorder such that beam breaking scatters randomly all over the sample. Clamping of the sample ends has the important consequence that the expansion of the system is hindered perpendicular to the direction of loading. This boundary condition promotes shear failure and leads to localization of the damage zone at an angle to the vertical prior to the development of the fault itself. Inside the shear band itself the high concentration of microcracks leads to fragmentation of the material into a large number of pieces. Figure 1(b) presents the final reassembled configuration where all particles are projected back to their original position after macroscopic failure has occurred in a sample of $10^{5}$ particles. The particles are colored according to the size of the fragment they belong to. The spatial distribution of damage is rather inhomogeneous in the sample: two big fragments can be observed reaching to the clamped boundaries of the sample, and the small fragments lie in between concentrated in a relatively narrow band. The stopping condition has the consequence that in some cases the relatively sharp wedge shaped end of the two biggest fragments breaks off and creates two more pieces which are still much larger than the rest of the fragments. However, the shear band is always easy to identify by visual inspection as a zone of contiguous fragmentation, as in Fig. 1(b). The damage zone is much bigger, containing smaller cracks and occasional loose fragments in a cloud around the main fault.

The spontaneous emergence of the shear band and its position, orientation, and structure are all important characteristics of the compressive failure of heterogeneous, disordered, or amorphous materials $[1-5,25]$. Since localization is preceded by random microcracking, the algorithmic determination of the position of the shear band is rather complex in DEM simulations. Although a large amount of distributed damage is obtained all over the sample, the damage is concentrated overwhelmingly in the shear band. A small number of single particles may also be liberated all over the sample [see Fig. 1(b)]. These only very rarely, if ever, form contiguous fragments of more than one particle. Accordingly the distribution of intermediate sized fragments (as opposed to loose fragments) is dominated by those in the shear band itself. Hence instead of analyzing the spatial positioning of broken beams, i.e., the zone of damage, we worked out an algorithm using information on the position and properties of the fragments inside the shear band itself.

To describe the position of the shear band we identify its central plane and characterize its position and orientation by its normal vector $\vec{n}_{d}$ and by a point $\vec{r}_{d}$ lying on it. The coordinates

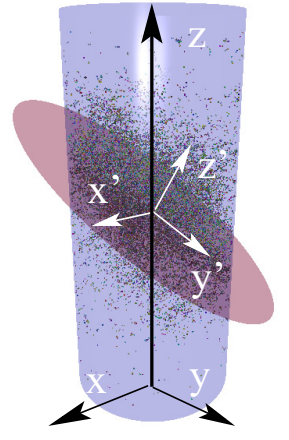

FIG. 2. Plane placed in the middle of the shear band. The black dots represent the center of mass position of fragments including those that are noncontiguous with the shear band. The figure also illustrates the coordinate systems used in the calculations. The origin of the $x, y, z$ coordinate system is the center of the bottom circle of the cylinder and the $z$ axis is directed along the cylinder axis. The $\left(x^{\prime}, y^{\prime}, z^{\prime}\right)$ system is centered at the support point of the plane and the $z^{\prime}$ axis is aligned with the normal vector $\vec{n}_{d}$ of the best fitting central fault plane.

of $\vec{r}_{d}$ are $\left(0,0, z_{d}\right)$ where the origin of the coordinate system is placed in the middle of the bottom circle of the cylinder with the $z$ axis pointing along the cylinder axis (see Fig. 2 for an illustration of the coordinate systems used in the calculations). As the first step of the algorithm the center of mass position of each fragment $\vec{r}_{i}^{f}, i=1, \ldots, K$ is determined, where $K$ is the total number of fragments in a sample. This point cloud is illustrated in Fig. 2, each dot representing a single fragment. The coordinate $z_{d}$ of the point fixing the position of the central plane is obtained as the $z$ coordinate of the center of mass position of the point cloud where each fragment has the same contribution to the averaging. Then we determined the polar angles $\omega_{i}$ and $\delta_{i}$ of the vectors $\vec{r}_{i}^{f d}=\vec{r}_{i}^{f}-\vec{r}_{d}$ with respect to the $x$ and $z$ axes of the coordinate system, respectively. After calculating the angle $\eta_{i}=\pi / 2-\delta_{i}$ for each fragment $i=$ $1, \ldots, K$, the discrete Fourier transform of the function $\eta(\omega)$ of the fragment ensemble was determined for each sample. The phase of the lowest order complex Fourier coefficient was used to identify the angular position $\phi$ of the damage plane with respect to the $x$ axis. Rotating the fragment cloud with this angle around the $z$ axis, its projection covers the smallest area on the $y-z$ plane. Looking at the system from the $x$ direction in this position the central fault plane forms a straight line. In order to identify the fault plane straight lines going through the center of mass $\vec{r}_{d}$ were fitted to the point cloud with the least square method subsequently removing those few points which have the largest distance from the line. Repeating the fitting and removing procedure with the rest of points after a few hundred iteration steps the algorithm provides the angle $\Theta$ of the central plane of the shear band with the load direction ( $z$ axis). The final result is illustrated in Fig. 2.

Due to the quenched structural disorder of the porous material the value of the angle $\Theta$ of single samples scatters over a range which depends on the system size. Figure 3 presents the probability distribution $p(\Theta, N)$ of the orientation angle $\Theta$ for all the system sizes $N$ considered. For the smallest system $N=10^{4}$ the distribution is quite broad; even high 


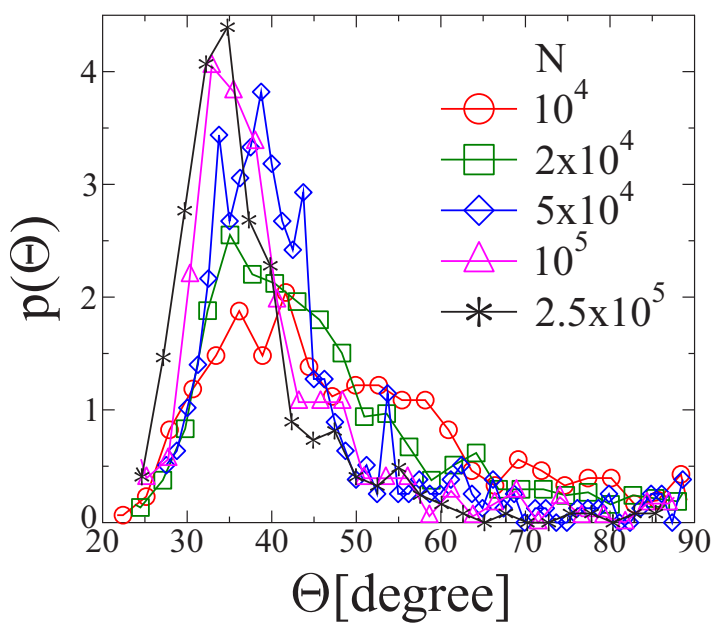

FIG. 3. Probability distribution $p(\Theta)$ of the angle $\Theta$ of the shear band with respect to the load direction (vertical direction in Fig. 1) for all system sizes $N$ considered. Increasing the system size the distributions get more and more peaked and their maximum gradually shifts to lower values.

angles $\Theta>50^{\circ}$ relatively often occur. The reason is that at this system size the width of the band is comparable to the cylinder diameter, which gives rise to a relatively homogeneous spatial distribution of fragments. Consequently, the plane orientation is sometimes identified to be close to horizontal. However, with increasing system size $N$ the band gets progressively more sharply defined; i.e., the distributions $p(\Theta, N)$ get more and more peaked and the position of the maximum shifts to progressively lower $\Theta$ values. For the largest system considerable probability is obtained only for angles in the range $25^{\circ}<\Theta<45^{\circ}$ with the most probable orientation of $\Theta \approx 34^{\circ}$. This variability, and the most likely value, are both quite similar to those seen in laboratory experiments, typically on samples 2 or more times greater in diameter [26]. In order to give a quantitative characterization of the sharpening effect of the system size, we performed a scaling analysis of the distributions obtained at different $N$ values assuming the finite size scaling form

$$
p(\Theta, N)=N^{\alpha} \Phi\left(\left[\Theta-\Theta_{c}(\infty)\right] N^{\alpha}\right)
$$

typical for critical phenomena [27,28]. Here $\Phi(x)$ denotes the scaling function and $\alpha$ is the scaling exponent. A very important outcome of the scaling analysis is the value of the central limit $\Theta_{c}(\infty)$ which denotes the orientation angle of the shear band of the infinite system where the shear band is sharply defined. By rescaling the distributions according to the scaling form Eq. (2) a good quality data collapse is obtained on Fig. 4. Due to normalization of the distributions the same scaling exponent is used along both axes. The best collapse is achieved with the parameter values $\alpha=0.25(5)$ and $\Theta_{c}(\infty)=29.8^{\circ}$ corresponding to an internal friction coefficient of 0.7 or so.

Of course, in the plane perpendicular to the load direction the system does not have any preferred direction for damaging; hence, the spatial distribution of microcracks and of the position of fragments should be nearly isotropic around the $z$ axis. It follows that the angle $\phi$ of the orientation of

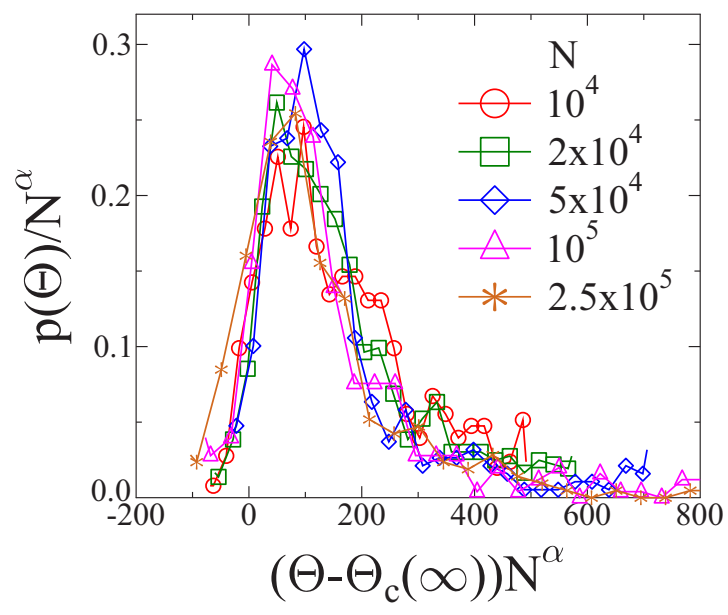

FIG. 4. Finite size scaling of the probability distribution $p(\Theta)$. Rescaling the two axes of Fig. 3 according to Eq. (2) a good quality data collapse is obtained.

the shear band is randomly selected so that its distribution should be uniform over the $-\pi \leqslant \phi \leqslant+\pi$ interval. This can be observed in Fig. 5 where the numerical results are well described by a sample obtained from a random uniform distribution for all $N$. This result confirms that the band orientation is isotropic around the load direction.

To quantify the thickness of the shear band for a given system size $N$ we shifted and rotated the samples such that the central planes coincide with each other. A local coordinate system $x^{\prime}, y^{\prime}, z^{\prime}$ is assigned to the point $\vec{r}_{d}$ such that the $x^{\prime}$ and $y^{\prime}$ axis fall in the central plane while $z^{\prime}$ is aligned with its normal vector (see Fig. 2 for an illustration). Then we calculated the probability distribution $p\left(z^{\prime}, N\right)$ of finding the center of mass position of fragments with a coordinate $z^{\prime}$. Figure 6 shows that for all system sizes the distributions $p\left(z^{\prime}, N\right)$ are well peaked and symmetric around $z^{\prime}=0$. The functional form of $p\left(z^{\prime}, N\right)$ also confirms the right selection of the central plane of the shear band. Careful analysis revealed that the curves of $p\left(z^{\prime}, N\right)$ cannot be fitted with a Gaussian; additionally Levy stable and

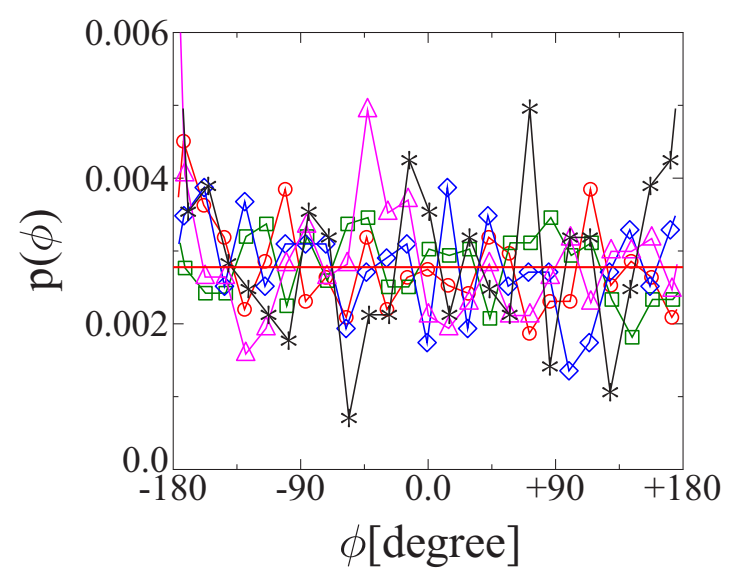

FIG. 5. Probability distribution of the angle $\phi$ of the shear band measured from the $x$ axis. The horizontal straight line represents the value $p(\phi)=1 / 2 \pi$ of the uniform distribution over the $-\pi \leqslant \phi \leqslant+\pi$ interval. The legend is the same as in Fig. 4. 


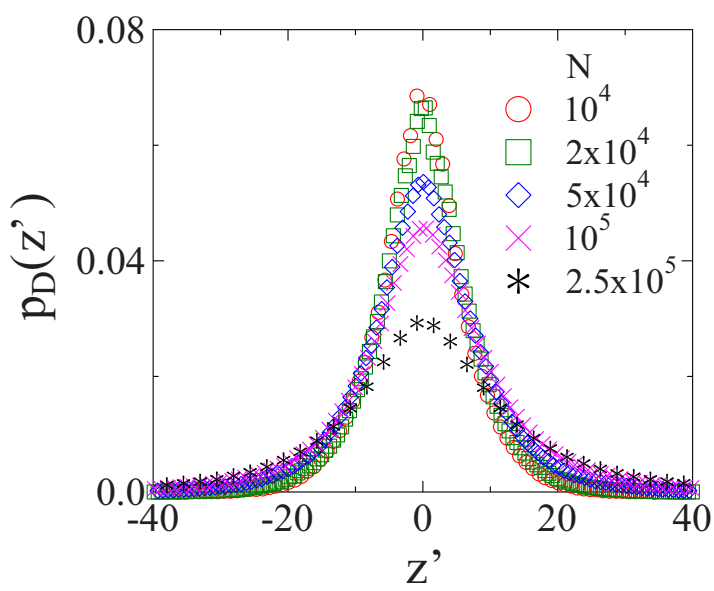

FIG. 6. Probability distribution of the coordinate $z^{\prime}$ of the center of mass of fragments for all system sizes $N$ considered.

Cauchy distributions were also tested, both giving relatively low quality fits in the tail region. The reason is that for such fat tailed distributions the system sizes we could achieve are still not sufficiently large. The results imply that fragments are well concentrated in the shear band, and hence, the band's thickness $W$ can be characterized by the width of the distribution. It can be seen in Fig. 6 that for larger samples the shear band gets broader in absolute terms. In order to analyze how $W$ depends on the system size, we carried out again finite size scaling of the distributions $p\left(z^{\prime}, N\right)$ assuming the scaling form

$$
p\left(z^{\prime}, N\right)=N^{-\gamma} \Psi\left(z^{\prime} / N^{\gamma}\right),
$$

where $\Psi(x)$ denotes the scaling function and $\gamma$ is the scaling exponent. In Fig. 7 a high quality data collapse is obtained with the exponent $\gamma=0.25(4)$. Deviations from the symmetric curve mainly occur for the smallest system size $N=10^{4}$, while the quality of scaling rapidly improves with increasing $N$. A very interesting consequence of the results is that the absolute width $W$ of the shear band increases as a power law

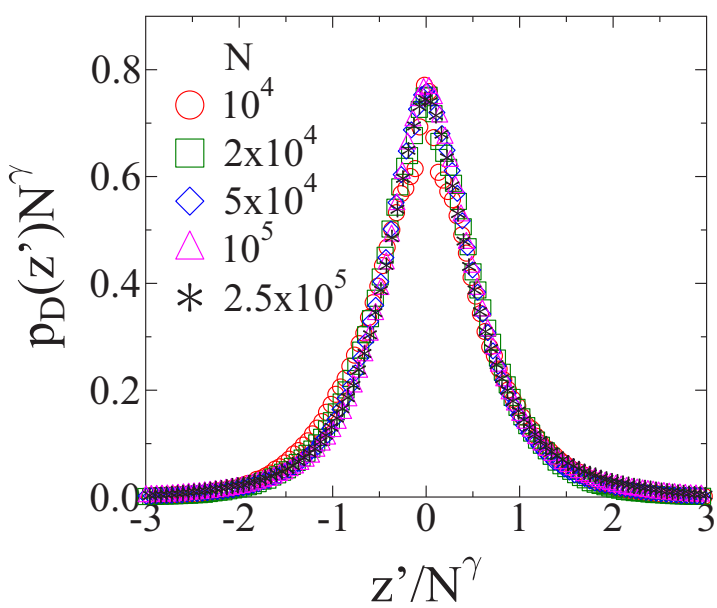

FIG. 7. Scaling plot of the probability distributions $p\left(z^{\prime}, N\right)$ obtained at different system sizes $N$. The value $\gamma=0.25$ of the scaling exponent provides the best data collapse. Deviations from the master curve can only be observed for the smallest system.
$W \sim N^{\gamma}$; however, the relative width $W / N^{1 / 3}$ decreases with the system size as

$$
\frac{W}{N^{1 / 3}}=N^{-(1 / 3-\gamma)},
$$

which shows that the band gets gradually sharper. Note that the two scaling exponents $\alpha$ and $\gamma$ practically coincide.

\section{STATISTICS AND GEOMETRY OF FRAGMENTS}

In a single sample we identified about 2000 and 30000 fragments in the contiguous shear band for the smallest and largest samples, respectively. Inside the shear band the sample gets gradually crushed so that the vast majority of the fragments are single particles, which play the role of fine powder in the system. These are unbreakable in the model since fracture can only take place at cohesive bonds between particles. In a real material individual grains can also fail by intragranular fracture, so the bandwidth of the size distribution can be even broader. Figure 1 shows a lateral observer's view of the surface of the sample shown after restoring the different fragments to their original place. Fragments of the largest four "intact" blocks are shown in blue. The fragments outside the zone of contiguous deformation are all single particles. In Fig. 8 we show a radial plot of the location of fragments composed of more than one particle, with respect to the cylinder axis, plotted as an integrated cross section. All of these fragments are located in the shear zone. Figures 1 and 8 confirm that the statistics of intermediate-sized fragments are dominated by those in the shear zone. The empty area in the middle of the cylinder in Fig. 8 indicates that the majority of fragments have only a single particle in that region. The size of fragments increases with the distance from the middle of the cylinder and the largest ones emerge on the surface of the sample. The reason is that pieces which break off the free

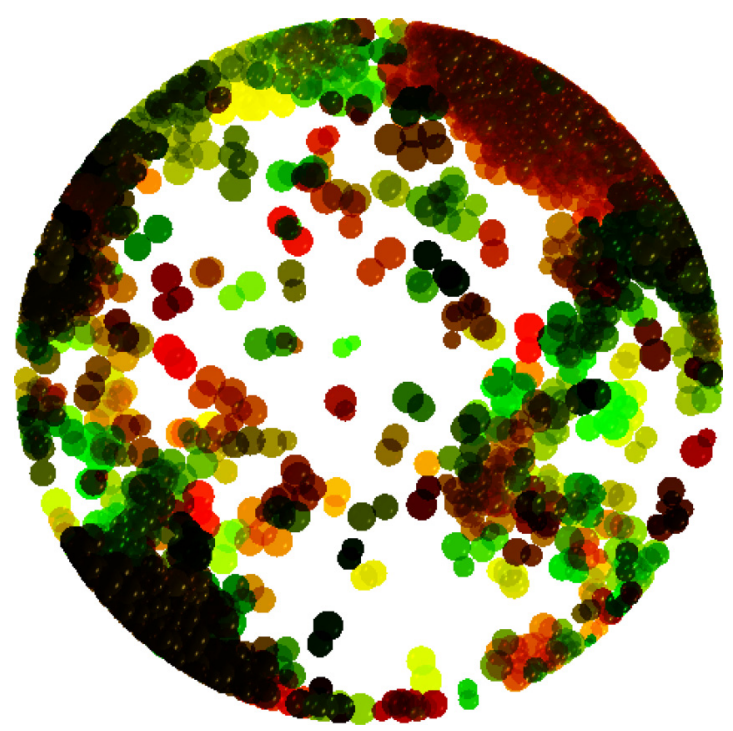

FIG. 8. Fragments comprising more than one particle in a sample of $N=10^{5}$ particles seen from the directions of the cylinder axes. The four largest fragments are not shown so that all the fragments of the figure fall inside the shear band. The color of fragments is randomly selected. 


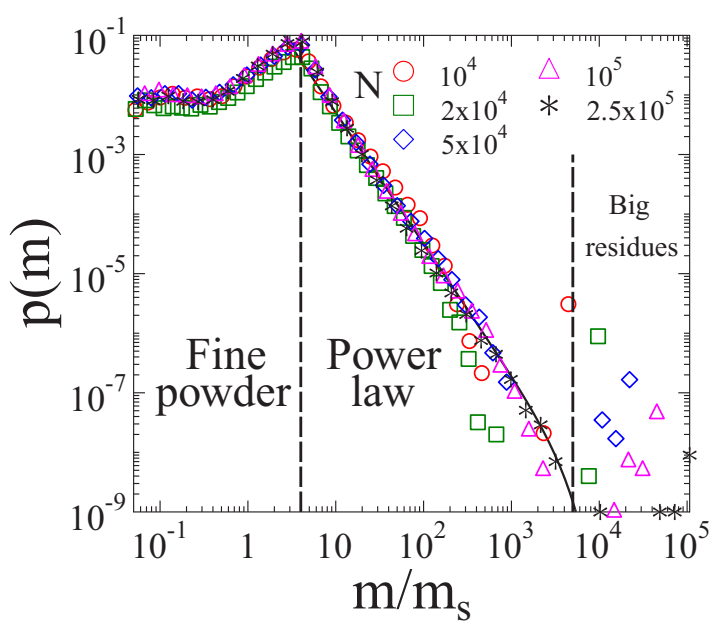

FIG. 9. Mass distribution of fragments for all sample sizes $N$. The power law regime is well separated from the fine powder and large residues. The bold line represents a fit with Eq. (5). The fragment mass is made dimensionless by dividing it with the average mass of single particles $m_{s}$.

surface or are formed close to the external surface can escape from the compressed body and do not suffer further crushing. This feature may not occur in natural samples, or those in the laboratory subject to a radial confining pressure.

The mass distribution $p(m)$ of all fragments is presented in Fig. 9 for different system sizes $N$. The single data points on the right end of the data sets represent the 2-4 largest pieces outside the contiguous shear band. The fine graded powder appears at the left side of the distributions where the functional form is determined by the mass distribution of single particles; hence it is the same for all cases. The most remarkable feature of the results is that fragments of intermediate sizes of the shear band have a power law mass distribution with a finite size cutoff of exponential shape. The distributions were fitted with the expression

$$
p(m) \sim m^{-\tau} \exp \left[-\left(m / m_{0}\right)^{c}\right]
$$

where the characteristic mass $m_{0}$ and the exponent $c$ only control the position and the precise shape of the cutoff. The results demonstrate in Fig. 5 that the exponent $\tau$ of the power law regime is the same for all cases $\tau=2.15$; the system size only affects the cutoff regime. Deviations are only observed for the smallest system. The best fit presented in Fig. 9 for $N=2.5 \times 10^{5}$ was obtained with the parameters $m_{0}=3140$ and $c=1.5$.

The power law functional form of fragment masses may indicate that during the compression process material regions inside the damage band undergo sequential breakup, gradually reducing their size. As fragments form they are confined between the two large residues connected to the clamped ends of the cylinder which hinders the expansion of the sample. Except for fragments which break off the sample along the cylinder surface, the majority of pieces undergo further breaking events. This hierarchical mechanism, similar conceptually to the geometric fragmentation model in Refs. [6,7], may explain why the exponent $\tau$ of our compression-induced fragmentation process is higher than its counterpart $\tau_{d}=1.9$

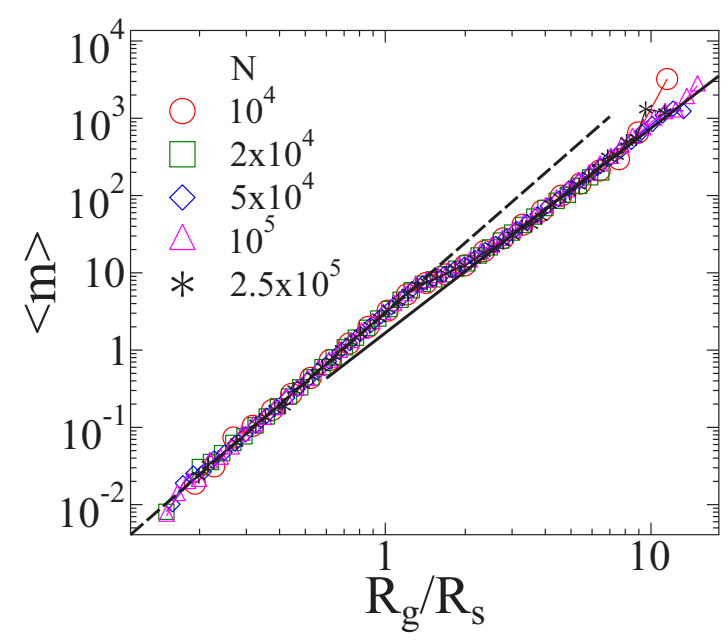

FIG. 10. Average mass of fragments as a function of the radius of gyration $R_{g}$ normalized by the average radius of single particles $R_{s}$. Results of the different system sizes fall on the top of each other. The two straight lines represent power laws of exponents 3 and 2.7.

obtained by dynamic fragmentation events induced, e.g., by projectile impact [29] or by impact with a hard plane [21,27].

Fragments observed in the fault gouge have been found to have a power law size distribution $N(l) \sim l^{-D}$, where $l$ denotes the length and $N(l)$ is the number of fragments of size greater than $l[7,11]$. Using the Euclidean or cubic relation of fragment mass and length $m \sim l^{3}$, the exponent $\tau$ of the probability density of fragment masses can be expressed in terms of $D$ as $\tau=1+D / 3$. Substituting the experimental value $D=2.6$, a corresponding mass exponent of $\tau=1.87$ is obtained. This is near but below $\tau=2.15$ inferred from the slope in Fig. 9. Some possible reasons for this systematic difference are explored below.

The cubic relationship between mass and length that we have assumed above holds for an ideal isotropic fragment. However, fragmentation occurs under compression and inside a confined subvolume of the sample. In this case one may expect that fragments may not have a completely isotropic shape. In order to obtain information about the geometrical features of fragments we determined their radius of gyration

$$
R_{g}^{2}=\frac{1}{n} \sum_{i=1}^{n}\left(\vec{r}_{i}-\vec{r}_{c}\right)^{2}
$$

where the sum runs over the particles of the fragment with position vectors $\vec{r}_{i}$, and $\vec{r}_{c}$ denotes the center of mass position of the fragment. Figure 10 presents the average mass $\langle m\rangle$ of fragments as a function of the radius of gyration for fragments consisting of more than 2 particles. Results of all system sizes fall on top of each other; just the upper cutoff of $R_{g}$ changes with $N$. Although the range of $R_{g}$ is rather limited, good quality power law behavior can be observed:

$$
\langle m\rangle \sim R_{g}^{D_{m}}
$$

It is very interesting that a crossover occurs between two regimes of different values of the exponent $D_{m}$ : for small 
fragments $D_{m}=3$ is obtained which demonstrates that fragments are space filling, compact objects. For large enough pieces a lower exponent $D_{m}=2.7$ emerges.

We now show that the lower exponent $D_{m}<3$ is a consequence of the self-affinity of fragment shapes. Let us assume that the shape of fragments can be approximated by cuboids of side length $S \leqslant I \leqslant L$. Then the radius of gyration can be expressed as

$$
R_{g}=\sqrt{S^{2}+I^{2}+L^{2}} / 2 \sqrt{3} .
$$

The shape of fragments can be characterized by the aspect ratios $S / L$ and $I / L$ which depend on the size of the object $R_{g}$. From Eq. (8) the extension $L$ of the fragment can simply be expressed in terms of the aspect ratios $S / L$ and $I / L$ and of $R_{g}$ as

$$
L=\frac{2 \sqrt{3} R_{g}}{\sqrt{(S / L)^{2}+(I / L)^{2}+1}},
$$

and hence, the volume of the fragment $V=S I L$ can be cast in the form

$$
V=\frac{3^{3 / 2} 8 R_{g}^{3}(S / L)(I / L)}{\left[(S / L)^{2}+(I / L)^{2}+1\right]^{3 / 2}} \sim R_{g}^{D_{m}} .
$$

On the right hand side we used the scaling form Eq. (7). For fragments which have a flat shape $S / L \ll 1$ and $I / L \ll 1$ hold so that the denominator falls close to one. In this case the aspect ratios can be expressed as a function of the radius of gyration

$$
\frac{S I}{L^{2}} \sim R_{g}^{-\left(3-D_{m}\right)}
$$

The result implies that the exponent $D_{m}<3$ is the fingerprint of the self-affine behavior of fragment shapes; i.e., large fragments have an anisotropic, slightly flat shape and as their size increases they get relatively flatter. This self-affinity is governed by the exponent $3-D_{m}$ which is the difference of the dimension of the embedding space $d=3$ and of the scaling exponent $D_{m}$ of Eq. (7). Similar self-affinity of fragment shapes has been found in the explosive fragmentation of shells [30]; however, for dynamic breakup of bulk materials the shape of pieces does not have this feature [31,32]. If we now use $D_{m}=2.7$ instead of $d=3$, such that $\tau=1+D / D_{m}$, with $D=2.6$ as observed experimentally or in natural fault gouge, then $\tau=1.96$, closer to our value of 2.15 from Fig. 9. The remaining discrepancy may be due to (a) absence of a radial confining pressure in the model, (b) the unbreakable nature of the discrete elements leading to a narrower bandwidth of fragment sizes, or (c) a different self-affinity exponent $D_{m}$ for natural fault gouges as a consequence of (b).

\section{DISCUSSION}

The compressive failure of rocks under slow compression is governed by the spontaneous emergence of a shear band where the high concentration of cracks gives rise to fragmentation into a large number of pieces. This fragmentation process and the resulting debris closely and quantitatively mimic the relevant mechanisms of the formation of the fault gouge on geological scales and in laboratory experiments. In the present paper we used discrete element simulations of the compressive failure of porous, disordered granular media to investigate the spatial structure of damage, the position and orientation of the shear band, and the statistics and geometry of its fragments.

Based on the spatial distribution of fragments we introduced an algorithm to determine the position and orientation of the shear band. Finite size scaling analysis showed that in the limit of large system sizes the central limit of the angle of the shear band to the maximum principal stress direction for an infinite sized system is 30 degrees, in good agreement with experiments. The width of the shear band proved to increase as a power law of the system size; however, the relative width decreases making the band sharper in larger systems.

Inside the shear band the gradual compression gives rise to fragmentation where the size of pieces spans a broad range. The majority of fragments falls into the powder phase comprising only single particles complemented by a few large residues attached to the loading plates. In the intermediate range the mass distribution of fragments has a power law functional form, where the exponent proved to be independent of the system size. The value of the exponent agrees well with those found for natural and laboratory-derived fault gouges. The hierarchical breakup of fragments induced by the slow compression may be responsible for the scale invariant fragment size. The confined geometry of the band imposes constraints on the shape of fragments: small fragments have an isotropic shape; however, the larger they become the more elongated shape they have. This self-affinity of fragment shapes can be quantified by a scaling exponent of the mass of fragments with respect to their radius of gyration.

Based on the simulations we conjecture that the tendency of larger particles to be flatter may explain the relative sharpening of the shear band with increasing sample size: the alignment of the larger particles is subparallel to the fault plane which inhibits rolling, which would tend to keep the band narrower for high aspect ratio particles. Large fragments tend to slide past each other or "shingle," like flat pebbles on a beach subject to water shear, which in turn favors a lower bandwidth.

For our DEM simulations porous rock samples were created by a single particle sedimentation algorithm. The size of sedimenting spheres was sampled from a log-normal distribution which well mimics the statistics of grain sizes in earth materials. The algorithm provides a high computational efficiency; however, it has the drawback that it imposes limitations on the available size range of grains. In the feasible range of the amount of disorder no relevant effect of the structure of the particle packing could be pointed out on the formation of the shear band. However, the sufficiently high sample to grain size ratio proved to be essential to obtain reliable quantitative results on the emergence, spatial structure, and fragment statistics of the damage band.

\section{ACKNOWLEDGMENTS}

We thank the projects K119967 and TAMOP-4.2.2.A11/1/KONV-2012-0036. This research was supported by the European Union and the State of Hungary, co-financed by the European Social Fund in the framework of the TAMOP4.2.4.A/2-11/1-2012-0001 National Excellence Program. 
[1] C. C. Hays, C. P. Kim, and W. L. Johnson, Phys. Rev. Lett. 84, 2901 (2000).

[2] H. Bei, S. Xie, and E. P. George, Phys. Rev. Lett. 96, 105503 (2006).

[3] D. Klaumünzer, A. Lazarev, R. Maaß, F. H. Dalla Torre, A. Vinogradov, and J. F. Löffler, Phys. Rev. Lett. 107, 185502 (2011).

[4] T. Unger, J. Török, J. Kertész, and D. E. Wolf, Phys. Rev. Lett. 92, 214301 (2004).

[5] M. L. Manning, E. G. Daub, J. S. Langer, and J. M. Carlson, Phys. Rev. E 79, 016110 (2009).

[6] D. L. Turcotte, Fractals and Chaos in Geology and Geophysics (Cambridge University Press, Cambridge, 1997).

[7] D. L. Turcotte, J. Geophys. Res. 91, 1921 (1986).

[8] I. Main, Rev. Geophys. 34, 433 (1996).

[9] J. A. Aström, Adv. Phys. 55, 247 (2006).

[10] C. A. Morrow and J. D. Byerlee, J. Struct. Geol. 11, 815 (1989).

[11] C. Marone and C. H. Scholz, J. Struct. Geol. 11, 799 (1989).

[12] S. J. Steacy and C. G. Sammis, Nature (London) 353, 250 (1991).

[13] J. A. Åström, H. J. Herrmann, and J. Timonen, Phys. Rev. Lett. 84, 638 (2000).

[14] S. Abe and K. Mair, Geophys. Res. Lett. 32, L05305 (2005).

[15] V. P. B. Esnault and J. N. Roux, Mech. Mater. 66, 88 (2013).

[16] A. Neveu, R. Artoni, P. Richard, and Y. Descantes, J. Mech. Phys. Solids 95, 308 (2016).

[17] K. Mair, I. Main, and S. Elphick, J. Struct. Geol. 22, 25 (2000).

[18] F. Kun, I. Varga, S. Lennartz-Sassinek, and I. G. Main, Phys. Rev. E 88, 062207 (2013).
[19] F. Kun, I. Varga, S. Lennartz-Sassinek, and I. G. Main, Phys. Rev. Lett. 112, 065501 (2014).

[20] G. Pál, F. Raischel, S. Lennartz-Sassinek, F. Kun, and I. G. Main, Phys. Rev. E 93, 033006 (2016).

[21] H. A. Carmona, F. K. Wittel, F. Kun, and H. J. Herrmann, Phys. Rev. E 77, 051302 (2008).

[22] T. Pöschel and T. Schwager, Computational Granular Dynamics (Springer, Berlin, 2005).

[23] P. K. Haff and B. T. Werner, Powder Technol. 48, 239 (1986).

[24] P. R. Sammonds, P. G. Meredith, and I. G. Main, Nature (London) 359, 228 (1992).

[25] Y. Shi, M. B. Katz, H. Li, and M. L. Falk, Phys. Rev. Lett. 98, 185505 (2007).

[26] M. S. Paterson and T.-F. Wong, Experimental Rock Deformation: The Brittle Field (Springer, Berlin, 1978).

[27] G. Timár, F. Kun, H. A. Carmona, and H. J. Herrmann, Phys. Rev. E 86, 016113 (2012).

[28] H. Nishimori and G. Ortiz, Elements of Phase Transitions and Critical Phenomena, 1st ed. (Oxford University Press, Oxford, 2011).

[29] G. Pál, I. Varga, and F. Kun, Phys. Rev. E 90, 062811 (2014).

[30] F. Kun, F. K. Wittel, H. J. Herrmann, B. H. Kröplin, and K. J. Maloy, Phys. Rev. Lett. 96, 025504 (2006).

[31] G. Domokos, F. Kun, A. A. Sipos, and T. Szabó, Sci. Rep. 5, 9147 (2015).

[32] D. D. Durda, A. C. Bagatin, R. A. Aleman, G. J. Flynn, M. M. Strait, A. N. Clayton, and E. B. Patmore, Planet. Space Sci. 107, 77 (2015). 